\title{
Diversidad y distribución de Araceae de la Reserva de la Biosfera Los Tuxtlas, Veracruz, México
}

\author{
Diversity and distribution of Araceae of the Reserva de la Biosfera Los Tuxtlas, Veracruz, \\ Mexico
}

\author{
Amparo Acebey ${ }^{1 *}$ y Thorsten Krömer $^{2}$ \\ ${ }^{1}$ Estación de Biología Tropical Los Tuxtlas, Instituto de Biología, Universidad Nacional Autónoma de México. Apartado postal 94, 95701, San Andrés \\ Tuxtla, Veracruz, México. \\ ${ }^{2}$ Centro de Investigaciones Tropicales, Universidad Veracruzana, interior de la ex hacienda Lucas Martín, Privada de Araucarias s/n, Col. 21 de Marzo, \\ 91019, Xalapa, Veracruz, México. \\ *Correspondencia: cacebey@gmx.net
}

Resumen. Este estudio documenta la diversidad, distribución altitudinal y geografía de Araceae en la región de Los Tuxtlas, Veracruz. Se escogió un transecto altitudinal en bosque tropical perennifolio y bosque mesófilo de montaña bien conservado desde los $100 \mathrm{~m}$ de altitud hasta la cima del volcán San Martín Tuxtla a los $1700 \mathrm{~m}$. En cada cota altitudinal de 100 m de elevación se realizaron muestreos en 3-4 parcelas de 400 m². El listado de aráceas presentado para la región de la Reserva de la Biosfera Los Tuxtlas se basa en datos propios, complementado con información de listas publicadas, bases de datos y revisión de herbarios. Se registraron 34 especies representadas en 9 géneros, de las cuales 5 son endémicas de México. Las aráceas (hemi-) epífitas representan aproximadamente el 11\% de la flora epifítica de la Estación de Biología Tropical Los Tuxtlas. A lo largo del gradiente altitudinal se observó un descenso constante del número de especies respecto al incremento de la altitud. El hallazgo de 3 nuevos registros de aráceas para la región de Los Tuxtlas y el estado de Veracruz demuestran que la realización de más inventarios florísticos sería importante.

Palabras clave: aráceas, bosque mesófilo de montaña, bosque tropical perennifolio, distribución geográfica, endemismo, epifitismo, gradiente altitudinal, riqueza de especies.

\begin{abstract}
This study documents the diversity, altitudinal and geographical distribution of Araceae in the region of Los Tuxtlas, Veracruz. An altitudinal transect was selected in mature tropical rain and cloud forest from $100 \mathrm{~m}$ elevation to the summit of the San Martín Tuxtla volcano at $1700 \mathrm{~m}$. In each range of $100 \mathrm{~m}$ elevation we carried out inventories of Araceae in 3-4 plots of $400 \mathrm{~m}^{2}$. The list of aroids presented for the region of the Reserva de la Biosfera Los Tuxtlas is based on our own data, supplemented with information from published sources, data bases and revision of herbarium specimens. We recorded a total of 34 species in 9 genera, of which 5 are endemic to Mexico. The (hemi-) epiphytic aroids represent about $11 \%$ of the epiphytic flora at the Biological Research Station Los Tuxtlas. A constant decline of the number of species was observed with increasing elevation along the altitudinal gradient. The discovery of 3 new records of aroid species for the region of Los Tuxtlas and the state of Veracruz shows that it would be important to carry out more floristic inventories.
\end{abstract}

Key words: aroids, cloud forest, elevational gradient, endemism, epiphytism, geographical distribution, species richness, tropical rain forest.

\section{Introducción}

La familia de plantas Araceae es principalmente tropical con una mayor diversidad de especies en Asia y América tropical (Croat, 1998). En México se han registrado 121 especies y 18 géneros, siendo los géneros Anthurium, Philodendron y Monstera los más numerosos en especies

Recibido: 22 marzo 2006; aceptado: 10 diciembre 2007
(Espejo y López-Ferrari, 1993). La mayoría de las especies se concentran en las zonas tropicales de los estados de Chiapas, Oaxaca y Veracruz, aunque existen algunos elementos de zonas templadas; por ejemplo, Arisaema (Bunting, 1965). El endemismo de aráceas en México es alto, principalmente en el género Anthurium en el cual de un total de 41 especies, 26 son endémicas (Croat, 1983, 1986); asimismo en Philodendron subgénero Philodendron de 21 especies, 7 son endémicas (Croat 1997). El estado de 
Veracruz alberga aproximadamente el $45 \%$ del total de las especies mexicanas (Sosa y Gómez-Pompa, 1994; T. B. Croat y A. Acebey, datos no publicados) y todavía presenta áreas poco exploradas, especialmente las que limitan con los estados de Oaxaca y Chiapas, por lo que se espera que dicho número se incremente. Tanto la lista florística de la Flora de Veracruz (Sosa y Gómez-Pompa, 1994) como el listado florístico electrónico de Los Tuxtlas (CastilloCampos et al., 2004) incluyen las Araceae, pero no aportan información adicional relevante sobre su distribución en municipios y elevaciones y el tipo de vegetación donde crecen u otros aspectos de su ecología.

En la región de Los Tuxtlas se encuentra el reducto de bosque tropical perennifolio más nórdico del continente, en el cual, junto con los otros tipos de vegetación presentes (p. ej. bosque mesófilo de montaña, bosque de pino y encino), han sido registradas un poco más de 3350 especies de plantas (Guevara et al., 2004). En las últimas décadas, tal riqueza de flora y fauna está amenazada por una continua deforestación y transformación del bosque natural en pasturas o plantaciones. En la región, la mayoría de los estudios florísticos se han realizado a altitudes bajas en los terrenos de la Estación de Biología Tropical Los Tuxtlas, registrando un total de 943 especies incluidas en 137 familias (Ibarra-Manríquez y Sinaca 1995, 1996a, 1996b, 1997; Riba y Pérez-García, 1997). Pocos investigadores, como Álvarez del Castillo (1977), han muestreado por arriba de los 900 metros. Con excepción del trabajo de Lira y Riba (1984), que estudiaron la diversidad y distribución altitudinal de las pteridófitas en el volcán Santa Marta, la mayor parte de los estudios se han enfocado en aspectos generales de la vegetación, como la composición florística, la estructura y la fisonomía.

Debido a que las aráceas son un elemento muy característico de la región de Los Tuxtlas, este estudio pretende documentar su diversidad de especies y analizar sus patrones de distribución altitudinal y geográfica.

\section{Material y métodos}

El estudio fue realizado entre abril y diciembre de 2005 en la Reserva de la Biosfera Los Tuxtlas (18 $05^{\prime}$ $\left.18^{\circ} 43^{\prime} \mathrm{N}, 94^{\circ} 35^{\prime}-95^{\circ} 25^{\prime} \mathrm{O}\right)$, en el sudeste del estado de Veracruz, México. En el área de estudio (Fig. 1) se escogió un transecto altitudinal en bosque tropical perennifolio (tropical rain forest) y bosque mesófilo de montaña (cloud forest) bien conservado, desde los $100 \mathrm{~m}$ de altitud, donde está ubicada la Estación de Biología Tropical Los Tuxtlas (EBT; con una área protegida de 640 ha), hasta la cima del volcán San Martín Tuxtla a los 1700 m snm. En cada cota altitudinal de $100 \mathrm{~m}$ se realizaron muestreos

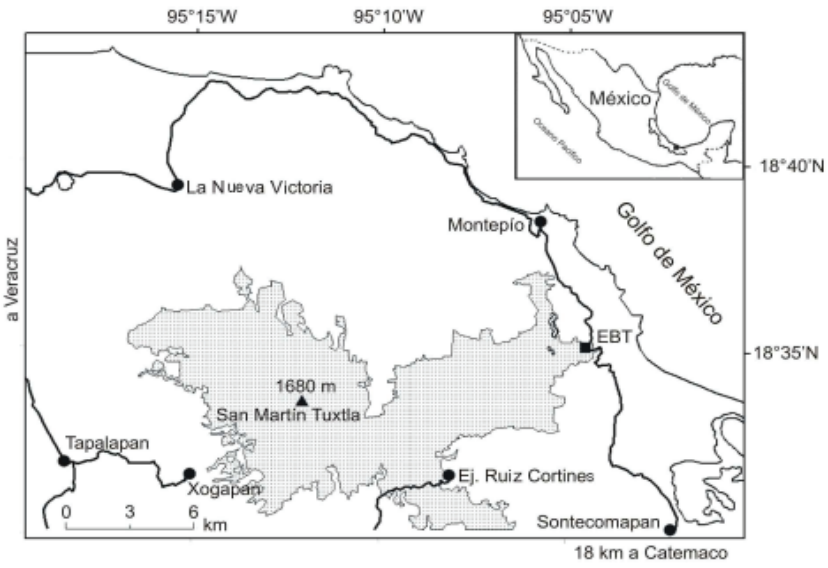

Figura 1. Mapa del área de estudio en la región noreste de Los Tuxtlas, Veracruz, México. El área sombreada corresponde al bosque tropical perennifolio bien conservado, en las laderas del volcán San Martín Tuxtla y en las 640 hectáreas de la Estación de Biología Tropical Los Tuxtlas (EBT).

de Araceae y de otros grupos selectos de plantas; por ejemplo, Bromeliaceae, Orchidaceae y varias familias de Pteridophyta, en 3-4 parcelas no permanentes de 400 $\mathrm{m}^{2}$ (total de 60 parcelas). La lista de las aráceas para la región de la Reserva de la Biosfera Los Tuxtlas basada en recolecciones y registros propios, se complementó con datos tomados de varias listas de especies (IbarraManríquez y Sinaca, 1996b; Ramírez, 1999; CastilloCampos et al., 2004). Además, se revisaron los herbarios de la Estación de Biología Los Tuxtlas y del Instituto de Ecología, A.C. con sus respectivas bases de datos. Los ejemplares recolectados durante el trabajo de campo fueron depositados en el Herbario Nacional de México (MEXU), con duplicados en el Herbario del Instituto de Ecología, A. C., Xalapa (XAL) y en el Missouri Botanical Garden, St. Louis (MO).

La vegetación dominante en la región de Los Tuxtlas corresponde originalmente a bosque tropical perennifolio (Rzedowski, 1978), pero el $80-90 \%$ fue destruida continuamente hasta el año 1990 (Dirzo y García, 1992). Actualmente, las laderas inferiores del volcán San Martín Tuxtla están cubiertas por un mosaico de vegetación dominado por potreros con árboles aislados y diferentes tipos de campos de cultivo, los cuales rodean los fragmentos de bosque remanentes. Entre las familias (géneros) de árboles más importantes están Leguminosae (Lonchocarpus spp.), Moraceae (Ficus spp.) y Lauraceae (Nectandra spp.). Entre los 1000 y $1550 \mathrm{~m}$ de altitud se encuentra un bosque mesófilo de montaña mayormente bien conservado, que contiene árboles de hasta $45 \mathrm{~m}$ de alto, tales como Ulmus mexicana (Liebm.) Planch., 
mientras que la cima está cubierta por un bosque enano de neblina (elfin forest) dominado por Oreopanax xalapensis (Kunth) Decne. et Planch. y Clusia salvinii Donn. Sm. Para una descripción más detallada de la vegetación de la región de Los Tuxtlas véase González Soriano et al. (1997) y Guevara et al. (2004).

\section{Resultados}

El listado de las aráceas de la Reserva de la Biosfera Los Tuxtlas incluye 34 especies en 9 géneros (Apéndice 1), 3 de las cuales son registros nuevos para el área de estudio. Los géneros Philodendron (7) y Anthurium (6) tienen el mayor número de especies, aunque Monstera (5) y Syngonium (4) también muestran un número de especies relativamente alto. Respecto a las diferentes formas de vida, un poco más de la mitad (18) de las especies son hemiepífitas trepadoras, mientras que 7 son terrestres, 4 epífitas y otras 4 epífitas-rupícolas o terrestres accidentales y sólo una es acuática libre flotadora. Las aráceas epífitas (incluyendo hemiepífitas trepadoras) representan aproximadamente el $11 \%$ de la flora epifítica de la EBT en relación al total de las epífitas registradas por IbarraManríquez et al. (1997) para esta zona.

La mayoría de las aráceas registradas para el área de estudio se distribuyen principalmente en tierras bajas, alcanzando altitudes intermedias hasta los $1100 \mathrm{~m}$ (Apéndice 1). A lo largo del gradiente altitudinal se observa un descenso constante y casi lineal del número de especies a medida que la altitud aumenta, mostrando una correlación negativa significativa $(r=-0,98, \mathrm{P}<0,01)$ entre la elevación y el número de especies (Fig. 2). Solamente Anthurium scandens (Aubl.) Engl. subsp. scandens, Monstera lechleriana Schott y Syngonium chiapense Matuda se encuentran a lo largo de todo el gradiente altitudinal.

En relación a su distribución geográfica, la mitad de

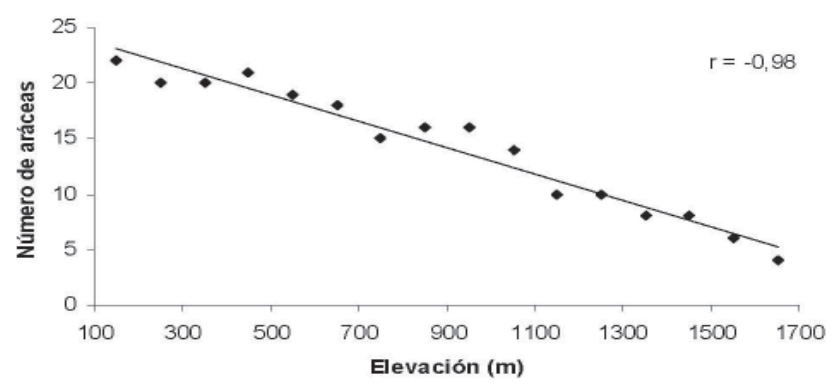

Figura 2. Correlación entre la elevación y el número de especies de aráceas en el bosque tropical perennifolio bien conservado en el área de estudio. las especies se distribuyen principalmente en México y Centroamérica, algunas incluso alcanzan el norte de Sudamérica (Apéndice 1). Otras 6 especies tienen una amplia distribución desde México hasta Ecuador o Bolivia, y sólo 1 es pantropical (Pistia stratiotes L.). Existen 6 taxa que sólo se encuentran en México, Guatemala y/o Belice, mientras que otros 4 son endémicas de México: Philodendron seguine subsp. lingua-bovis Grayum, Spathiphyllum cochlerispathum (Liebm.) Engl., Syngonium neglectum Schott.

\section{Discusión}

La contribución de las aráceas al total de la riqueza de especies en la región de Los Tuxtlas es limitada; sin embargo, representa casi un $65 \%$ de las aproximadamente 53 especies de esta familia registradas para el estado de Veracruz (T. B. Croat y A. Acebey, datos no publicados). En la EBT las aráceas representan un porcentaje relativamente bajo $(11 \%)$ de la flora epifítica, lo cual probablemente se relaciona con el hecho de encontrarse en el bosque tropical perennifolio más septentrional del Neotrópico. Este valor coincide con las estimaciones hechas por Wolf y Flamenco (2003) (11\%) para el estado de Chiapas en un intervalo altitudinal de 0 a $500 \mathrm{~m}$. El porcentaje de las aráceas epífitas puede alcanzar valores mayores al $20 \%$ en latitudes cercanas al ecuador (Cuadro 1), donde regiones con alta precipitación, tales como Tiputini en Ecuador y Río Caquetá en Colombia, presentaron los valores más altos (Benavides et al., 2005; Kreft et al., 2004). No obstante, la proporción de aráceas en relación al total de epífitas depende fuertemente del tamaño del área de estudio. Así, Krömer et al. (2005) encontraron que la familia puede representar hasta el $40 \%$ de la flora epifitica en un área de apenas $400 \mathrm{~m}^{2}$ en un bosque submontano de Bolivia.

La diversidad de las aráceas a lo largo del gradiente altitudinal en el área de estudio muestra un patrón similar al encontrado para el estado de Chiapas en México y para algunos países andinos en Sudamérica, en donde el número total de especies de aráceas o su contribución al total de la flora epifitica disminuye linealmente con el incremento de la altitud (Ibisch et al., 1996; Kessler, 2002; Wolf y Flamenco, 2003; Krömer et al., 2005). Al contrario de las aráceas, las orquídeas y los helechos epifitos muestran una correlación positiva con el incremento de la altitud hacia altitudes intermedias, en donde alcanzan su mayor diversidad (Krömer et al., 2005). En bosques tropicales perennifolios de baja elevación las aráceas epífitas pueden alcanzar la misma riqueza de especies que las orquídeas y ser aún más numerosas que los helechos (Wolf y Flamenco, 2003), mientras que normalmente por 
Cuadro 1. Número de especies y proporción de aráceas en relación al total de la flora epifítica para varias localidades neotropicales de tierras bajas entre 0-500 m snm, con datos de la precipitación anual.

\begin{tabular}{lccccccc}
\hline & $\begin{array}{c}\text { BCI, } \\
\text { Panama }^{1} \\
2750 \mathrm{~mm}\end{array}$ & $\begin{array}{c}\text { La Selva, } \\
\text { Costa Rica } \\
4000 \mathrm{~mm}\end{array}$ & $\begin{array}{c}\text { Suromoni, } \\
\text { Venezuela }^{3} \\
2700 \mathrm{~mm}\end{array}$ & $\begin{array}{c}\text { Madidi, } \\
\text { Bolivia }^{4} \\
>2000 \mathrm{~mm}\end{array}$ & $\begin{array}{c}\text { Tiputini, } \\
\text { Ecuador }^{5} \\
3200 \mathrm{~mm}\end{array}$ & $\begin{array}{c}\text { Rio Caquetá, } \\
\text { Colombia }^{6} \\
3060 \mathrm{~mm}\end{array}$ & $\begin{array}{c}\text { EBT, } \\
\text { México }^{7} \\
>4000 \mathrm{~mm}\end{array}$ \\
\hline $\begin{array}{l}\text { Número de } \\
\text { aráceas }\end{array}$ & 30 & 76 & 14 & 21 & 70 & 56 & 18 \\
$\begin{array}{l}\text { Total de } \\
\text { epífitas }\end{array}$ & 216 & 368 & 53 & 147 & 313 & 213 & 162 \\
$\begin{array}{l}\text { Porcentaje de } \\
\text { la flora }\end{array}$ & 13.9 & 20.7 & 26.4 & 14.3 & 22.4 & 26.3 & 11.1
\end{tabular}

Datos obtenidos de:

${ }^{1}$ Croat (1978), ${ }^{2}$ McDade et al. (1994), ${ }^{3}$ Engwald (1999), ${ }^{4}$ Acebey y Krömer (2001), ${ }^{5}$ Kreft et al. (2004), ${ }^{6}$ Benavides et al. (2005), 7 este estudio (solo EBT)

encima de elevaciones medias la diversidad de Araceae es más reducida. Mayo et al. (1997) mencionan que su crecimiento depende de la abundante disponibilidad de agua y humedad atmosférica, ya que no se encuentran bien adaptadas, ni estructural ni fisiológicamente, a condiciones áridas y frías. Por esta razón, su diversidad es mayor en tierras bajas y húmedas cercanas al ecuador y sólo algunas especies con adaptaciones especiales, como por ejemplo las geófitas, toleran ambientes extremos.

El muestreo intensivo de aráceas realizado en el área de estudio condujo a 3 registros nuevos, 1 para la región de Los Tuxtlas, Philodendron standleyi Grayum, y 2 para el estado de Veracruz, Xanthosoma wendlandii (Schott) Schott, especie anteriormente conocida sólo de otros estados de México (Espejo y López-Ferrari, 1993), y Spathiphyllum brevirostre (Liebm.), Schott, considerada inicialmente como una especie nueva (T. B. Croat, com. pers.). Esta última es una especie endémica de México, poco común y sólo conocida para la región de Los Tuxtlas, Veracruz, y gracias a este proyecto fue posible recolectar material adicional para confirmar su identidad.

Anthurium andicola Liebm., A. lucens Standley ex Yuncker y A. verapazense Engl. sólo fueron registradas de la sierra de Santa Marta, la cual aparentemente tiene elementos florísticos distintos a los presentes en el área del volcán San Martín Tuxtla. Esta última situación la registran Krömer y Acebey (2007) para las bromeliáceas y también se puede observar en el caso de las pteridófitas del volcán Santa Marta (Lira y Riba, 1984), al compararlos con los datos del volcán San Martín Tuxtla (T. Krömer y A. Acebey, datos no publicados). Entre las posibles causas para explicar esta situación, se encuentra la heterogeneidad de los bosques tropicales perennifolios de ambas zonas, así como los efectos de clima, topografía y suelos específicos de cada una de las áreas mencionadas.

Este estudio permitió también aclarar la identidad de algunas especies que aún se encontraban erróneamente identificadas en varios herbarios, incluyendo MEXU y XAL, a pesar de que en los listados florísticos se las citaba correctamente. Este es el caso de Dieffenbachia oerstedii Schott, determinada como Dieffenbachia seguine (Jacq.) Schott, especie originaria de las Antillas y de la región amazónica de Sudamérica, pero ampliamente cultivada como ornamental en los trópicos de América (Croat, 2004). Philodendron seguine subsp. lingua-bovis determinada equivocadamente como P. guttiferum Kunth, taxon originario de la región amazónica de Sudamérica. Grayum (1996), señala que $P$. seguine subsp. lingua-bovis es endémica de Veracruz y la zona fronteriza con Oaxaca. Por último, cabe señalar que Philodendron scandens K. Koch et Sello es en realidad un sinónimo de $P$. hederaceum (Jacq.) Schott (Croat, 1997). Por otra parte, existe una especie de Monstera poco conocida en la región de Los Tuxtlas, la cual es frecuente que se confunda con Monstera acuminata K. Koch y cuya identidad taxonómica todavía es motivo de discusión. Espejo y López-Ferrari (1993) y Sosa y Gómez Pompa (1994), la enlistan como Monstera lechleriana, un taxón muy variable que probablemente incluya varias especies y que tiene un origen sudamericano. Sin embargo, M. egregia Schott citada por Madison (1977) como sinónimo de la anterior, originalmente fue descrita con material mexicano, por esta razón, los especialistas en la familia están considerando retomar el nombre de M. egregia para las poblaciones de México y países vecinos (T. B. Croat, com. pers.). De esta manera, una vez publicado este cambio la especie Monstera egregia pasaría a reemplazar todo el material hasta ahora determinado como M. lechleriana.

Los 3 nuevos registros de aráceas encontrados para la región de Los Tuxtlas y el estado de Veracruz demuestran que la realización de nuevos inventarios, a elevaciones mayores y en lugares menos explorados es 
todavía importante. Asimismo, el estudio más detallado de familias o grupos ricos en epífitas, que por otra parte están generalmente poco representados en colecciones de herbario, es fundamental para conocer más acerca de su diversidad, su ecología y su aporte a la flora en general.

\section{Agradecimientos}

A Thomas B. Croat, por su ayuda en la identificación de las especies y sus acertados comentarios; a Adolfo Espejo, Ana Rosa López-Ferrari, Michael Kessler, y a 2 revisores anónimos, por la revisión del manuscrito y sus valiosos aportes al mismo. A Francisco G. Lorea por proveer la base de datos de Araceae y acceso al los ejemplares del Herbario XAL, a Claudia Gallardo por las fotografías de casos dudosos. Este estudio fue financiado con una beca posdoctoral otorgada a Thorsten Krömer por la Universidad Nacional Autónoma de México.

\section{Literatura citada}

Acebey, A. y T. Krömer. 2001. Diversidad y distribución vertical de epífitas en los alrededores del campamento río Eslabón y de la laguna Chalalán, Parque Nacional Madidi, Dpto. La Paz, Bolivia. Revista de la Sociedad Boliviana de Botánica 3:104-123.

Álvarez del Castillo, G. C. 1977. Estudio ecológico y florístico del cráter del volcán San Martín Tuxtla, Ver., México. Biotica 2:3-54.

Benavides, A. M. D., A. J. M. Duque, J. F. Duivenvoorden, G. A. Vasco y R. Callejas. 2005. A first quantitative census of vascular epiphytes in rain forest of Colombian Amazonia. Biodiversity and Conservation 14:739-758.

Bunting, G. S. 1965. Commentary on Mexican Araceae. Gentes Herbarum 9:291-382.

Castillo-Campos, G., S. Guevara y G. Sánchez-Ríos. 2004. Listado florístico electrónico de Los Tuxtlas, Versión 1.0. Flora de Veracruz. Instituto de Ecología, Xalapa, Veracruz.

Croat, T. B. 1978. Flora of Barro Colorado Island. Stanford University Press, California. 943 p.

Croat, T. B. 1983. A revision of the genus Anthurium (Araceae) of Mexico and Central America, part 1: Mexico and Middle America. Annals of the Missouri Botanical Garden 70:211417.

Croat, T. B. 1986. The distribution of Anthurium (Araceae) in Mexico Middle America and Panama. Selbyana 9:94-99.

Croat, T. B. 1997. A revision of Philodendron subgenus Philodendron (Araceae) for Mexico and Central America. Annals of the Missouri Botanical Garden 84:311-704.

Croat, T. B. 1998. Tropical aroids: taxonomy, diversity and ecology. In diversity and taxonomy of tropical flowering plants, P. Mathew y M. Sivadasan (eds.). Mentor, Calicut (India) p. 235-286.

Croat, T. B. 2004. Revision of Dieffenbachia (Araceae) of
Mexico, Central America, and the West Indies. Annals of the Missouri Botanical Garden 91:668-772.

Dirzo, R. y M. C. García. 1992. Rates of deforestation in Los Tuxtlas, a neotropical area in southeast Mexico. Conservation Biology 6:84-90.

Engwald, S. 1999. Diversität und Ökologie der vaskulären Epiphyten eines Berg- und eines Tieflandregenwaldes in Venezuela. Books on Demand, Hamburg. 390 p.

Espejo S. A. y A. R. López-Ferrari. 1993. Las monocotiledóneas mexicanas, una sinopsis florística. 1. Lista de referencia, parte II. Anthericaceae, Araceae, Arecaceae, Asparagaceae, Asphodelaceae y Asteliaceae. Comisión Nacional para el Conocimiento y Uso de la Biodiversidad y Universidad Autónoma-Metropolitana, Iztapalapa, México, D.F. 70 p.

González Soriano, E., R. Dirzo y R. C. Vogt (eds.) 1997. Historia natural de Los Tuxtlas. Instituto de Biología, Universidad Nacional Autónoma de México, México D.F. 647 p.

Grayum, M. H. 1996. Revision of Philodendron subgenus Pteromischum (Araceae) for Pacific and Caribbean tropical America. Systematic Botany Monographs 47:1-233.

Guevara, S., J. Laborde y G. Sánchez (eds.). 2004. Los Tuxtlas. El paisaje de la sierra. Instituto de Ecología, Xalapa, Veracruz. $287 \mathrm{p}$.

Ibarra-Manríquez, G. y S. Sinaca Colín. 1995. Lista florística comentada de la Estación de Biología Tropical "Los Tuxtlas", Veracruz, México. Revista de Biología Tropical 43:75-115.

Ibarra-Manríquez, G. y S. Sinaca Colín. 1996a. Estación de Biología Tropical "Los Tuxtlas", Veracruz, México: Lista florística comentada (Mimosaceae a Verbenaceae). Revista de Biología Tropical 44:41-60.

Ibarra-Manríquez, G. y S. Sinaca Colín. 1996b. Lista comentada de plantas de la Estación de Biología Tropical "Los Tuxtlas", Veracruz, México: (Violaceae-Zingiberaceae). Revista de Biología Tropical 44:427-447.

Ibarra-Manríquez, G. y S. Sinaca. 1997. Lista florística de Los Tuxtlas. Fanerógamas. In Historia natural de Los Tuxtlas, E. González Soriano, R. Dirzo y R. C. Vogt (eds.). Instituto de Biología, Universidad Nacional Autónoma de México, México D.F. p. 162-174.

Ibarra-Manríquez, G., M. Martínez-Ramos, R. Dirzo y J. NúñezFarfán. 1997. La vegetación. In Historia natural de Los Tuxtlas, E. González Soriano, R. Dirzo y R. C. Vogt (eds.). Instituto de Biología, Universidad Nacional Autónoma de México, México D.F. p. 61-82.

Ibisch, P. L., A. Boegner, J. Nieder y W. Barthlott. 1996. How diverse are neotropical epiphytes? An analysis based on the "Catalogue of flowering plants and gymnosperms of Peru". Ecotropica 2:13-28.

Kessler, M. 2002. The elevational gradient of Andean plant endemism: varying influences of taxon-specific traits and topography at different taxonomic levels. Journal of Biogeography 29:1159-1165.

Kreft, H., N. Köster, W. Küper, J. Nieder y W. Barthlott. 2004. Diversity and biogeography of vascular epiphytes in western Amazonia, Yasuní, Ecuador. Journal of Biogeography 31:1463-1476.

Krömer, T., M. Kessler, S. R. Gradstein y A. Acebey. 2005. 
Diversity patterns of vascular epiphytes along an elevational gradient in the Andes. Journal of Biogeography 32:17991809.

Krömer, T. y A. Acebey. 2007. The bromeliad flora of the San Martín Tuxtla volcano, Veracruz, Mexico. Journal of the Bromeliad Society 57:62-69.

Lira, S. R. y R. Riba. 1984. Aspectos fitogeográficos y ecológicos de la flora pteridológica de la Sierra de Santa Marta, Veracruz, México. Biotica 9:451-467.

Madison, M. 1977. A revision of Monstera (Araceae). Contributions from the Gray Herbarium 207:3-100.

Mayo, S. J., J. Bogner y P. C. Boyce. 1997. The genera of Araceae. Royal Botanical Gardens, Kew. 370 p.

McDade, L. A., K. S. Bawa, H. A. Hespenheide y G. S. Hartshorn. 1994. La Selva: ecology and natural history of a neotropical rain forest. The University of Chicago Press, Illinois.486 p.
Ramírez, F. 1999. Flora y vegetación de la sierra de Santa Marta, Veracruz. Tesis, Facultad de Ciencias, Universidad Nacional Autónoma de México. México, D.F. 409 p.

Rzedowski, J. 1978. Vegetación de México. Limusa, México D.F. $431 \mathrm{p}$.

Riba, R. y B. Pérez-García 1997. Lista florística de Los Tuxtlas. Pteridofitas. In Historia natural de Los Tuxtlas, E. González Soriano, R. Dirzo y R. C. Vogt (eds.). Instituto de Biología, Universidad Nacional Autónoma de México, México D.F. p. 175-181.

Sosa, V. y A. Gómez-Pompa. 1994. Araceae. In Flora de Veracruz-Lista Florística. Instituto de Ecología, Xalapa, Veracruz y University of California, Riverside. p. 27-29.

Wolf, J. H. D. y A. Flamenco. 2003. Patterns in species richness and distribution of vascular epiphytes in Chiapas, Mexico. Journal of Biogeography 30:1689-1707.

Apéndice 1. Listado de las aráceas presentes en la Reserva de la Biosfera Los Tuxtlas con datos acerca de la forma de vida (acu: acuática, ep: epífita, hep: hemiepífita, rup: rupícola, ter: terrestre), intervalo altitudinal en Los Tuxtlas, intervalo altitudinal y distribución en el Neotrópico (MEX: México, CRI: Costa Rica, GTM: Guatemala, BLZ: Belice, HND: Honduras, NIC: Nicaragua, PAN: Panamá, ANT: Antillas, BOL: Bolivia, COL: Colombia, ECU: Ecuador, BRA: Brasil, GUF: Guyana Francesa, VEN: Venezuela).

\begin{tabular}{|c|c|c|c|c|}
\hline Especies de aráceas & $\begin{array}{l}\text { Forma de } \\
\text { vida }\end{array}$ & $\begin{array}{l}\text { Intervalo altitudinal } \\
\text { Los Tuxtlas }(m)^{l}\end{array}$ & $\begin{array}{l}\text { Intervalo altitudinal } \\
\text { Neotrópicos }(m)^{2}\end{array}$ & $\begin{array}{c}\text { Distribución en el } \\
\text { Neotrópico }^{3}\end{array}$ \\
\hline Anthurium andicola Liebm. & ep, rup & 1490 & $1000-2500$ & MEX, GTM \\
\hline A. flexile Schott subsp. flexile & hep & $100-700$ & $0-1000$ & MEX-PAN \\
\hline $\begin{array}{l}\text { A. flexile subsp. muelleri (J.F. } \\
\text { Macbride) Croat et R.A. Baker }\end{array}$ & hep & $800-1700$ & $1000-1700$ & MEX, GTM \\
\hline A. lucens Standl. ex Yuncker & ep, rup & $600-950$ & $100-2000$ & MEX-HND \\
\hline $\begin{array}{l}\text { A. pedatoradiatum Schott subsp. } \\
\text { pedatoradiatum }\end{array}$ & ter & $100-200$ & $0-800$ & MEX, GTM \\
\hline $\begin{array}{l}\text { A. pentaphyllum (Aubl) G. Don var. } \\
\text { bombacifolium (Schott) Madison }\end{array}$ & hep & $100-700$ & $0-800$ & MEX-PAN \\
\hline $\begin{array}{l}\text { A. scandens (Aubl.) Engl. subsp. } \\
\text { scandens }\end{array}$ & ep & $100-1700$ & $0-2700$ & $\begin{array}{l}\text { MEX-BOL, BRA, } \\
\text { ANT }\end{array}$ \\
\hline $\begin{array}{l}\text { A. schlechtendalii Kunth subsp. } \\
\text { schlechtendalii }\end{array}$ & ep, (ter) & $100-1100$ & $0-1000(-1600)$ & MEX-HND \\
\hline A. verapazense Engl. & ep, (ter) & $1100-1400$ & $100-800(-1400)$ & MEX, BLZ, GTM \\
\hline Dieffenbachia oerstedii Schott & ter & $100-1000$ & $0-1260$ & MEX-PAN \\
\hline Dieffenbachia wendlandii Schott & ter & $100-200$ & $75-900$ & MEX-PAN \\
\hline Monstera acuminata K. Koch & ep & $100-1100$ & $0-800$ & MEX-HND \\
\hline M. deliciosa Liebm. & hep & $700-1700$ & $400-2000$ & MEX-PAN \\
\hline M. lechleriana Schott & hep & $100-1600$ & $0-2100$ & MEX-BOL \\
\hline M. punctulata (Schott) Engl. & ep & $400-700$ & $100-1500(-1900)$ & $\begin{array}{l}\text { MEX, GTM, CRI, } \\
\text { PAN }\end{array}$ \\
\hline $\begin{array}{l}\text { M. tuberculata Lundell var. } \\
\text { tuberculata }\end{array}$ & ep & $100-500$ & $0-500$ & MEX, BLZ, GTM \\
\hline
\end{tabular}


P. hederaceum (Jacq.) Schott var.

P. inaequilaterum Liebm. subsp. inaequilaterum

$P$. radiatum Schott var. radiatum

P. sagittifolium Liebm.

P. seguine Schott subsp. linguabovis Grayum

P. standleyi Grayum*

P. tripartitum (Jacq.) Schott

Pistia stratiotes L.

Rhodospatha wendlandii Schott var. wendlandii

Schott**

S. cochlearispathum (Liebm.) Engl.

S. ortgiesi Regel

Syngonium angustatum Schott

S. chiapense Matuda

S. neglectum Schott

S. podophyllum Schott var. podophyllum

Xanthosoma robustum Schott

$X$. wendlandii (Schott) Schott** hederaceum

Spathiphyllum brevirostre (Liebm.)

\begin{tabular}{|c|c|c|c|}
\hline hep & $100-1100$ & $0-1200(-1500)$ & $\begin{array}{l}\text { MEX-BOL, ANT, } \\
\text { BRA, VEN, Guayanas }\end{array}$ \\
\hline hep & $100-1100$ & $0-1500(-1800)$ & $\begin{array}{l}\text { MEX-COL, VEN, } \\
\text { ECU }\end{array}$ \\
\hline hep & $100-500$ & $0-700$ & MEX-COL \\
\hline hep & $100-1100$ & $0-1800$ & MEX-COL \\
\hline hep & $100-1300$ & $0-1100(-1400)$ & MEX, endémica \\
\hline hep & $900-1500$ & $(400-) 600-1800(-2100)$ & MEX-PAN \\
\hline hep & $100-1300$ & $0-1300(-1500)$ & MEX-ECU \\
\hline acu & $0-500$ & $0-1260$ & Pantropical \\
\hline hep & $100-600$ & $0-550(-1600)$ & MEX-COL, VEN \\
\hline hep & $1100-1600$ & (580) $1000-2000$ & MEX, endémica \\
\hline ter & $100-200^{\S}$ & $0-1600$ & MEX, endémica \\
\hline ter & $100-1000$ & $0-900(-1500)$ & MEX, HND \\
\hline hep & $100-200^{\S}$ & $0-700(-1200)$ & MEX-CRI \\
\hline hep & $100-1700$ & $0-1200(-1500)$ & MEX, GTM \\
\hline hep & $600-700^{\S}$ & (0-) 350-1 700 & MEX, endémica \\
\hline hep & $100-1500$ & $0-1000(1500)$ & $\begin{array}{l}\text { MEX-BOL, BRA, } \\
\text { GUF }\end{array}$ \\
\hline ter & $100-900$ & $0-1400$ & MEX, CRI \\
\hline ter & $100-200$ & $0-1300$ & MEX-PAN, VEN \\
\hline
\end{tabular}

* Registro nuevo para la región de Los Tuxtlas; ** registro nuevo para el estado de Veracruz; ${ }^{1}$ datos obtenidos en el presente estudio; ${ }^{2}$ T. B. Croat, datos no publicados; ${ }^{3}$ base de datos Tropicos (www.tropicos.org); ${ }^{\$}$ observada sólo en vegetación secundaria fuera del bosque tropical perennifolio bien conservado. En negrillas, especies registradas en la Estación de Biología Tropical Los Tuxtlas. 